\title{
Analysis of Famous TCM Doctor Zan Qiang's Experience in the Treatment of Perimenopausal Osteoporosis
}

\author{
Zengrui Zhang ${ }^{1}$, Bin Shao ${ }^{2}$, Yanzhao Li ${ }^{1}$, Qiang Zan ${ }^{1} 3^{*}$ \\ ${ }^{1}$ Shaanxi University of Traditional Chinese Medicine, Xianyang 712046, China \\ ${ }^{2}$ Binzhou Medical University Hospital, Binzhou 256603, China \\ ${ }^{3}$ Affiliated Hospital of Shaanxi University of Traditional Chinese Medicine, Xianyang 712000, China
}

\begin{abstract}
The purpose of this study was to analyze the clinical experience of Zan Qiang, a famous doctor of Traditional Chinese Medicine in Shaanxi province, in the treatment of perimenopausal osteoporosis. Zan believed that the main etiology and pathogenesis of perimenopausal osteoporosis were "deficiency of kidney essence, decreased marrow and bone", "deficiency of liver and kidney" and "stasis caused by deficiency". Although it is a chronic physiological disease, and the pathological process is irreversible, the treatment groups of "tonifying kidney and filling essence", "tonifying liver and tonifying kidney" and "tonifying deficiency and promoting blood circulation" can significantly improve the symptoms of patients, slow down the progress of the disease and improve the quality of life of patients.
\end{abstract}

Key words: ZAN Qiang; Liver and kidney treatment; Perimenopausal osteoporosis

Publication date: May, 2021; Publication online: 31 May, 2021

*Corresponding author: Qiang Zan, zanqiang2009@163.com

\section{Introduction}

Perimenopause is the course of normal physiological changes that begin in a woman's mid-40s and continue for 12 months after menopause. Perimenopausal osteoporosis is due to women in the perimenopausal period, ovarian function gradually decline, estrogen level is lower than before, osteolysis, increased bone resorption, resulting in bone mineral content decreased, cavity formation, increased fragility ${ }^{[1]}$. The clinical symptoms are mostly systemic drowsiness, lumbar and back pain and diffuse bone pain all over the body, which increases the probability of fracture. In epidemiology, the prevalence of osteoporosis in perimenopausal women is high, and the incidence has an increasing trend with the increase of age ${ }^{[2]}$. The mainstream treatment of modern medicine is estrogen replacement therapy, but its disadvantages are more obvious, it will increase the incidence of invasive breast cancer, endometrial cancer and cardiovascular and cerebrovascular events, has certain limitations in the treatment of this disease. In traditional Chinese medicine, most of the drugs used in the treatment of osteoporosis at this stage are drugs to fill up deficiency, which have few side effects and significant curative effects, and have unique advantages in the cognition and treatment of this disease ${ }^{[3]}$. Therefore, perimenopausal osteoporosis is the dominant disease under the treatment of traditional Chinese medicine. Osteoporosis in perimenopausal period belongs to the category of "impotence" and "various diseases before and after menopause" in Chinese medicine. Zan Qiang, a famous Chinese medicine veteran, has been engaged in clinical and scientific research work for more than 30 years, and has rich experience and unique insights on the treatment of osteoporosis in this stage. It was also the author's honor to learn from Mr. Zan Qiang. 


\section{Etiology and pathogenesis analysis of perimenopausal osteoporosis}

\subsection{Deficiency of kidney essence, reduced marrow and dry bone}

Traditional Chinese medicine believes that the kidney is congenital, the main bone, health marrow, according to the $\mathrm{Su}$ Wen - Wei Lun, in which it is stated: "the kidney has evil heat, hot turbidity essence dry, resulting in marrow reduction and bone dry, lumbar spine can not move, become bone impotence." "Kidney can hide water, now this water defeated by the fire, causing bone dry and marrow deficiency, as well as bone impotence." Kidney essence boom or decline depends on wethear skeleton is strong or not. If kidney essence is sufficient, marrow is active and filled inside bone, which makes the skeleton strong; If kidney eseence is insufficient, the source of marrow is cut, causing empty inside the bone, which makes the bone deficient. Deng Linwen ${ }^{[4]}$, through the study of the distribution pattern, found that kidney deficiency type was the most common of osteoporosis syndrome type. Therefore, Teacher Zan believes that kidney essence deficiency is the basic pathogenesis of perimenopausal osteoporosis, and the treatment of perimenopausal osteoporosis should focus on tonifying kidney.

\subsection{Deficiency of liver and kidney as well as blood and essence}

In the theory of five elements theory of TCM, the kidney is water organ and the liver is wood organ. Water can nourish wood. Kidney essence deficiency causing liver displacement. According to modern medicine, the incidence of perimenopausal osteoporosis is the significant decrease in the level of estrogen, the activation of osteoclast signaling pathway, osteolysis, decreased bone mass, and the formation of cavity. Therefore, perimenopausal osteoporosis is a natural process of female physiological degeneration. Ye Tianshi expounded the concept of "women are born with liver" in Lin Zheng Zhi Nan Yi An, which also emphasized the importance of liver in the process of female growth and development. So in the treatment, it is necessary to pay attention to the liver blood while filling the kidney essence.

While reinforcing the liver and blood, iver should be smoothed and qi should be regulated. Women in the perimenopausal period are not only significantly more likely to suffer from depression than ordinary people, but also have more serious symptoms than ordinary people ${ }^{[5]}$. In the theory of traditional Chinese medicine, liver and blood deficiency for a long time affects the physiological function of the liver, leading to the failure of qi, liver qi stagnation, and depression and liver depression are closely related. Thus, this syndrome in the two deficiency of liver and kidney on the basis of the disease continues to progress. So Teacher Zan believes that the symptoms of a long illness are liver stagnation and qi stagnation, as blood and essence is full, it is harmony with the liver qi.

\subsection{Stasis caused by deficiency}

Perimenopausal osteoporosis patients are mainly composed of those with essence and blood deficiency, which leads to deficiency of vital energy and weak promotion, and blood deficiency leads to loss of vascular surplus and loss nourish of bone. Ren Zhiqiang ${ }^{[6]}$ studied on the relationship between blood stasis and bone metabolism in 64 patients with primary osteoporosis and found that blood stasis could cause abnormal bone metabolism and lead to osteoporosis. Sui chengzhi ${ }^{[7]}$, according to the hemorheology index, found that the blood stasis of 132 perimenopausal women was scored quantitatively. It was also found that the blood stasis degree of perimenopausal women was positively correlated with age. Stasis block caused the pulse path not passable which leads to pain. Therefore, this stage of osteoporosis patients with liver and kidney deficiency usually are having blood stasis. So, Mr. Zan teaches us that when treating of perimenopausal osteoporosis, the concept of "organic wholeness and treatment should be used based on syndrome differentiation".

\section{Syndrome differentiation and treatment}

\subsection{Tonifying kidney and filling essence}

At the early stage of the disease, the symptoms are mostly acid and soft waist and knee, drowsiness, yin deficiency, night sweat, dark red tongue and thin pulse. For this kind of patients who lack of kidney essence and bone marrow, Teacher Zan often use "Du Qi Wan" as the basic prescription with the tortoise plastron, antler gum, epimedium, psoralea, eucommia ulmoides, radix achyranthes, mulberry parasitic adding in for enhancing the efficacy. Du Qi Wan is made based on the liu-wei-di-huang pill added with schisandra for nourishing kidney yin and takes its convergence solid astringent work to solid essence. In the prescription, the rtoise plastron and antler gum -- a kind of animal products, helps supplement the kidney essence. Solitary yin doesn't develop, and yang can't last long, so epimedium and 
psoralea are added to warm the kidney and help yang, in order to generate the power of yin and yang, and to help transform yin essence. Eucommia ulmoides, achyranthes bidentata, mulberry parasitic work to strengthen waist and knee, muscles and bones. At the same time, in modern pharmacological studies, mulberry parasitic promotes the expression of OPG protein, reduces the level of inflammatory factor IL-1, reduces the level of OT and ALP in serum, and improves the negative balance of bone metabolism, so as to play a role in combating osteoporosis. Epimedium can not only enhance the function of osteoblasts, but also inhibit the generation and mechanism of osteoclasts $^{[8]}$. The whole prescription is based on tonifying yin essence of kidney, and assisted by tonifying yang, and given consideration to relieving waist and knee pain. For patients with osteoporosis in the early perimenopausal period, this formula has achieved good effects.

\subsection{Tonifying the liver and the kidney}

Every time the patient appears with former syndromes, such as pale face and mouth, "jin" deficiency, stiff hands and feet, Mr. Zan, basis on the front prescription, added white peony root, angelica belonging to the liver meridian, to supplement the liver and blood. If patients are suffering depression, fullness and discomfort in chest and hypochondrium, $\mathrm{Mr}$. Zan teachers to use the Yi Guan Jian to nourish the liver and kidney, coursing liver qi and resolving the depression, and a little pericarpium citri reticulatae viride which always help to smooth liver qi, plus the mulberry parasitism, eucommia bark, achyranthes taken for it's strengthening effect on waist and knee, as well as bones and muscles, with white peony root, dogwood, dodder, milkvetch seed added into this liver and kidney yin medicine, to relieve painful symptoms, meanwhile smooth qi and calm mood.

\subsection{Supplement deficiency and promote blood circulation}

Long deficiency of liver and kidney causes stasis of qi and blood. In replenishing deficiency and blood circulation, Teacher Zan thinks that excessive circulation rhythm of blood can smooth the veins, relieve the pain, and the effect is quick, but can cause loss of vital qi, which makes the weak patients weaker. Therefore, Teacher Zan adds a little blood activating drugs in the basis of removing stasis not to break blood and Qi for relieving symptoms but dose not harm vital qi. Therefore, in the treatment of perimenopausal osteoporosis for patients, on the basis of tonifying the liver and benefiting the kidney, these drugs are added that angelica, motherwort, rose flowers and other mild blood-activating drugs. And the study showed that leonurus alkaloid can inhibit the generation of osteoclasts ${ }^{[9]}$. Supplementing the kidney essence as well as the liver blood, smoothing qi and blood, are Mr. Zan's three principles of medication for the treatment of perimenopausal osteoporosis, and the three principles complement each other in the clinical practice.

\section{A case report}

Patient Sun, female, 50 years old, was first diagnosed at 10:00 on April 23, 2019. Main complaint: Waist and knee pain for 1 month, aggravated with activity limitation for 10 days. History of the disease: The patient developed pain and discomfort in the waist and knee without obvious inducement 1 month ago, which worsened after fatigue. After working 10 days ago, he felt worse with lumbago, stooping down and turning around, and had difficulty in getting up and turning over. No significant improvement was found after rest. At beginning, it also occurred that low mood, easily agitated, hot flashes, night sweat, tinnitus, insomnia and more dreams. Tongue dark red, thin moss, Menstrual history: Beginning age: 14 years old, 3-5d/26-45d, Ending age: 49 years old. Auxiliary examination: serum FSH $41.2 \mathrm{mIU} / \mathrm{ml}$, serum $\mathrm{E}_{2} 9.6 \mathrm{pg} / \mathrm{ml}$, blood calcium tendency for 2.3 / L, blood phosphorus tendency for 1.1 / L, collagen type I carboxyl end peptide beta special sequence (beta CTX) $0.58 \mathrm{ng} / \mathrm{ml}$, bone mineral density (BMD) value is -2.4 ( lumbar vertebra $1-4$ ). The radiograph of the lumbar spine showed disc herniation at L4/L5 and degenerative bone lesions of the vertebral body. Diagnosis: perimenopausal osteoporosis, TCM diagnosis: bone dysfunction (kidney deficiency and liver stagnation syndrome). Treatment: tonifying kidney and dredging liver. Recipe: radix rehmanniae $30 \mathrm{~g}$, radix ophiopogonis $10 \mathrm{~g}$, melia toosendan $5 \mathrm{~g}$, qingpi $5 \mathrm{~g}$, Chinese wolfberry $15 \mathrm{~g}$, angelica $10 \mathrm{~g}$, cuscuta $10 \mathrm{~g}$, comvetch $10 \mathrm{~g}$. Seven doses, applying $1200 \mathrm{ml}$ water to boil until $500 \mathrm{ml}$ left, then remove the residue, and take warm decoction twice one day, respectively in the morning and evening.

The second visit(on May 8, 2019): The patient complained of a slightly reduced sense of fatigue in the waist, limitation of activity, and obvious improvement in symptoms of mood, night sweats, insomnia and dreaminess, pink tongue, and heavy and thin pulse. The symptoms of yin 
deficiency and liver stagnation were well improved in the patient. Therefore, on the basis of anterior treatment, $15 \mathrm{~g}$ of mulberry parasitum, $15 \mathrm{~g}$ of epimedium, and $10 \mathrm{~g}$ of yujin were added in seven doses. The decocting and taking method were the same as before.

The third visit (on May 23, 2019): the patient's waist pain was significantly relieved, with free movement, more flexible bending and squatting, and stable mood. The tongue is pale red with thin white coating and a fine pulse. The patient was asked to take guilu erxian gum to consolidate the effect of tonifying kidney and supplementing essence, and have appropriate physical exercises for adjusting the mood.

\section{Conclusion}

In recent years, the incidence of perimenopausal osteoporosis patients is high coupled with the limitations of modern medicine in the treatment of osteoporosis patients in this period, the use of traditional Chinese medicine theory treatment effect is better. Mr. Zan Qiang, in diagnosis and treatment of perimenopausal osteoporosis, collects thoughts from various academic schools, on the basis of inheriting the essence of the sages, combining with years of clinical experience, put forward, with idea of liver and kidney treatment concept as basis, according to the course of continuous progress, during treatment, it should be taken that "the overall concept and symptoms that not only tonifying deficiency, but also giving attention to qi stagnation and blood stasis symptoms, respectively applying the treatment principles that "filling kidney essence", "filling liver and kidney" and "tonifying deficiency qi and promoting blood circulation". The paper proposes a new traditional Chinese medicine idea for the treatment of perimenopausal osteoporosis.

\section{References}

[1] Zhang Y. Study on the relationship between estrogen level and osteoporosis in perimenopausal period[J]. Technology Wind,2020(12):260-261.

[2] Lin XS, Wang HY, Xiao QH, Han J, Liu HC, Liu HJ. An epidemiological study of bone mass loss in 717 perimenopausal women[J]. Chinese Journal of Osteoporosis,2017,23(03):363-367.

[3] Yang ZD. Research progress of treating osteoporosis with traditional Chinese medicine[J]. System Medicine,2019,4(04):193-195.

[4] Deng LW, Mu L, Liu Y. Distribution of TCM syndromes in 130 cases of postmenopausal osteoporosis[J].Journal of Chengdu University of Traditional Chinese Medicine,2016,39(02):76-78.

[5] de Kruif M, Spijker AT, Molendijk ML. Depression during the perimenopause: A meta-analysis. J Affect Disord. 2016;206:174-180. doi:10.1016/j.jad.2016.07.040

[6] Ren ZQ, Yan XX, Jin DX, Zhuang H, Ding JY, Ma H. Study on the relationship between blood stasis and bone metabolism in primary osteoporosis[J]. China Journal of Traditional Chinese Medicine and Pharmacy,2015,30(05):1838-1840.

[7] Sui CZ, Liu ZK, Gan WD, Chen SQ, Fan XJ, Lin ZY, Chen Q. Study on the correlation between bone metabolism and "blood stasis" in perimenopausal women[J]. Chinese Journal of Osteoporosis,2016,22(11):1418-1424.

[8] Zhang X, Liu T, Huang YL, Wismeijer D, Liu YL. Icariin: Does it have an osteoinductive potential for bone tissue engineering?[J]. Phytotherapy Research,2014,28(4).

[9] Zhang Y, Tian KM. Effect and mechanism of leonurine hydrochloride on osteoclast formation[J]. Journal of Modern Medicine \& Health,2018,34(20):3215-3217. 